\title{
Article
}

\section{Laves phase formation in high entropy alloys}

\author{
Roman Ryltsev 1,2®, Svetlana Estemirova ${ }^{1,2}$, Evgenii Sterkhov ${ }^{2}$, Lubov Cherepanova ${ }^{1}$, Denis Yagodin ${ }^{1}$, Nikolay \\ Chtchelkatchev ${ }^{2,3}$, Nikolay Dubinin ${ }^{1,2}$, and Sergey Uporov ${ }^{1,2}$ (D) \\ 1 Institute of Metallurgy, Ural Branch of Russian Academy of Sciences, Amundsena str. 101, 620016 \\ Ekaterinburg, Russia \\ 2 Ural Federal University, Mira str. 19, 620002 Ekaterinburg, Russia \\ 3 Vereshchagin Institute for High Pressure Physics, Russian Academy of Sciences, 108840 Troitsk, Moscow, \\ Russia \\ * Correspondence: segga@bk.ru; Tel.: +7-908-919-29-99
}

\begin{abstract}
One of the intriguing recent results in the field of high-entropy alloys is the discovery of single-phase equiatomic multi-component Laves intermetallics. However, there is no clear understanding that a combination of chemical elements will form such high-entropy compounds. Here we contribute to understanding this issue by modifying the composition of duodenary TiZrHfNbVCrMoMnFeCoNiAl (12x) alloy in which we recently reported the fabrication of hexagonal C14 Laves phase. We consider three alloys based on 12x: $7 x=12 x-\mathrm{VCrMoMnFe}, 12 \mathrm{x}+\mathrm{Sc}, 12 \mathrm{x}+\mathrm{Be}$ and observe that all of them crystalize with the formation of $\mathrm{C} 14$ Laves phase as a dominant structure. We report that $12 x+$ Be alloy reveals single-phase $\mathrm{C} 14$ structure with very high concentration of structural defects and ultra-fine dendritic microstructure with almost homogenous distribution of the constituted elements over the alloy matrix. The $7 \mathrm{x}$ and $12 \mathrm{x}+\mathrm{Sc}$ alloys contain $\mathrm{C} 14$ as a main phase and unknown impurity phases. To characterize the materials, we examine their heat capacity, electrical conductivity and magnetic properties. The measurements reveal that the Laves phases are Curie-Weiss paramagnets, which demonstrate metallic conduction; $7 x$ and $12 x$ alloys also reveal a pronounced Kondo-like anomaly. Analysis of experimental data as well as ab initio calculations suggests that chemical complexity and compositional disorder cause strong $s-d$ band scattering and thus the rather high density of $d$-states in the conduction band. Analysis of the results suggests that the mechanism of Laves phase formation in multicomponent multi-principal element metallic alloys is may be the same as in polydisperse hard-spheres mixtures. Another important conclusion is that the configurational entropy is a negligible factor in the stabilization of multi-element Laves phases.
\end{abstract}

Keywords: high-entropy alloy; Laves phase; microstructure; electrical conductivity; magnetization; density of states; $a b$ initio calculations.

\section{Introduction}

One of the widely accepted paradigms in modern materials science is the use of multicomponent multi-principal element alloys to design new materials [1-6]. The terms high-entropy alloys (HEAs), complex concentrated alloys, and compositionally complex alloys are used to name such materials.

Over the past 15 years of intensive studies of HEAs, the researches have been focused on simple solid solutions (SSSs), such as FCC, BCC, HCP, and their mixtures. Special attention has been given to synthesize single-phase SSSs and study their properties and phase stability. To date, only several tens of single-phase high-entropy SSSs are known [7]. Unfortunately, they usually do not demonstrate any exceptional properties and are unable to compete with traditional functional materials.

This situation inspires searching for new types of HEAs. One of the promising classes includes multicomponent intermetallic compounds whose structure is more 
complex than for SSS HEAs. In particular, single-phase metallic HEAs with B2 structure [8,9] and half-Heusler type structure [10] have been fabricated. There is also a broad separate field of high-entropy oxides, which is also under active development [11]. Following Refs. [8,9], we will further refer to such systems as high-entropy intermetallic compounds (HEIC), although the role of the entropy in their formation is a matter of discussion [7].

One of the most intriguing recent results in the field of HEIC is the fabrication of equiatomic multi-component Laves intermetallics. In particular, single-phase C14 hexagonal structures have been successfully synthesized in several equiatomic alloys with the number of components from 4 to 28 [12-16,16,17]. Recently, we have made another step on this way by fabricating stable C14 Laves phase in duodenary TiZrHfNbVCrMoMnFeCoNiAl alloy [18]. We will further refer to such compounds as high-entropy Laves phases (HELPs). The duodenary HELP, which we recently reported, will be specially referred to as $12 x$.

The discovery of HELPs arise several fundamental issues. First, the mechanisms responsible for Laves phase formation in multicomponent alloys are not clear. To explain Laves phase stability, a number of simple geometric or electronic characteristics are usually utilized $[14,15,19]$. Among them are atomic size ratio, atomic size polydispersity $(\delta)$, valence electron concentration (VEC), electronegativity differences $(\Delta \chi)$, etc. However, there are at least two general problems that restrict strongly the prognostic power of these characteristics. First, their ranges, which are empirically obtained to be optimal for Laves phase formation, are rather broad and can include many alloys that do not form that phases. For example, atomic size ratios which are typically observed for binary Laves phases are within a range of 1.05 to 1.67 [20]. On the one hand, all the reported HELPs satisfy this criterion. On the other hand, there are many elements whose sizes are within this ratio, and not each equiatomic combination of these elements will form Laves phases. A similar situation takes place for other simple characteristics. Second, a selectivity of these characteristics decreases with an increase the number of components. For example, if we compare VECs for a $\mathrm{N}$-nary and $(\mathrm{N}+1)$-ry equiatomic alloys containing transition metals then the results may depend weakly on the choice of the additional element in the case of $N \gg 1$. Thus, VEC can fail to estimate if the addition of some element in multicomponent alloy will destabilize the Laves phase.

It seems that some complex interplay between geometric factors, electronic structure, configurational entropy, and maybe other factors is responsible for HELPs stability. Therefore, there is no intuitive understanding that a combination of chemical elements will form HEPLs. Here we contribute to understanding this issue. We consider a series of alloys by extracting/adding elements from the $12 x$ alloy. Thus, we study the following systems: TiZrHfNbCoNiAl (7x), $12 x+$ Sc, $12 x+$ Be. The first one is $7 x=12 x-$ VCrMoMnFe which means we extract from the base alloy a series of neighboring $3 \mathrm{~d}$ metals $(\mathrm{V}, \mathrm{Cr}, \mathrm{Mn}, \mathrm{Fe})$ as well as one $4 \mathrm{~d}$ metal $(\mathrm{Mo})$. This extraction changes substantially both the distribution of interparticle distances (geometric factor) and electronic characteristics and so it is interesting if this alloy will also form HELP. The motivation to consider $12 x+S c$ and $12 x+$ Be is that we add to the base $12 x$ alloy the elements with either larger $(\mathrm{Sc})$ or smaller size $(\mathrm{Be})$ to check if they destabilize the parent Laves phase.

Another issue is the physicochemical properties of HELPs, which are important for possible practical applications of such systems. So far, the researches have been focused on the structure and phase stability of fabricated HELPs and therefore their characteristics are not well understood. To fill partially this gap, we address the thermodynamic, electronic, and magnetic properties of the alloys under investigation.

\section{Materials and Methods}

The compounds were prepared of pure elemental metals (>99.99\%) by arc-melting under a flowing helium atmosphere. The alloy ingots were inverted and re-melted at least ten times to attain chemical and structural homogeneity. The mass loss after 

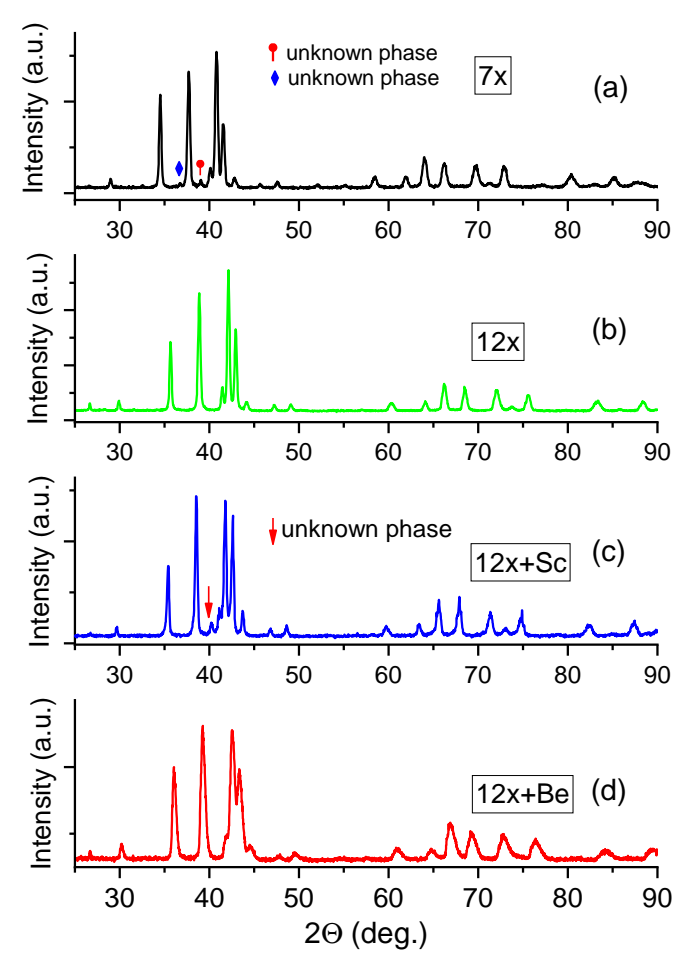

Figure 1. X-ray diffraction spectra of annealed alloys under consideration.

melting was less than $0.3 \%$. Homogenization of the samples was performed by vacuum annealing at the temperature of $673 \mathrm{~K}$ for $50 \mathrm{~h}$. X-ray diffraction analysis (XRD) was carried out using a Shimadzu XRD-7000 diffractometer with CuKff-radiation, Ni filter, and graphite monochromator in a range of angles of $15-150^{\circ}$ with a step $\Delta(2 \theta)=0.03^{\circ}$ and $4 \mathrm{~s}$ exposure at each step. A certified silicon powder was used as an external standard. The structural parameters were calculated using the GSAS software [21]. The parameters of the microstructure (the average value of coherent scattering regions (CSR) and the average value of microdeformation) were determined by the broadening of the diffraction lines using the graphical method of the Williamson-Hall [22]. Microstructure of the compounds was examined by both scanning electron microscopy (SEM) and optical microscopy methods by using a LOMO MMU-3 metallurgical microscope equipped with a CCD SONY ICX452AQ camera and a Carl Zeiss EVO 40 instrument, respectively. The samples for optical micrographs were previously mechanically polished and then chemically etched in a solution of $\mathrm{HNO}_{3}: \mathrm{HF}: \mathrm{H}_{2} \mathrm{O}$ taken in the ratio of 1:3:7. The electron microscopy analysis was performed on mechanically polished samples, without additional chemical etching. Specific heat of the samples was measured on a DSC 214 Polyma calorimeter in the temperature range of $100-520 \mathrm{~K}$. Magnetization (by vibrating sample magnetometry) and electrical conductivity (by four probe method) of the compound were explored using a Cryogenic CFS-9T-CVTI measurement system in a temperature interval of $300-5 \mathrm{~K}$. The resolution for magnetization and conductivity tests was 5 and $0.5 \%$, respectively.

\section{Results}

\subsection{X-ray diffraction analysis}

Powder X-ray diffraction patterns of the alloys under investigation are shown in Fig. 1. Analysis of the data reveals that $12 x$ and $12 x+B e$ alloys are single-phase systems in which all diffraction lines can be assigned to a hexagonal C14 Laves phase (prototype $\mathrm{MgZn}_{2}$ ). Most of XRD peaks in $7 \mathrm{x}$ and $12 \mathrm{x}+\mathrm{Sc}$ alloys also belong to C14 symmetry but there are a few undetectable peaks and so these alloys are not single-phase systems. It is hardly possible to bring these peaks into correlation with some structures because the 
Table 1: The structural parameters and the average values of microstrain $(\varepsilon)$ for the annealed samples of TiZrHfNbCoNiAl (7x), TiZrHfNbVCrMoMnFeCoNiAl (12x), TiZrHfNbVCrMoMnFeCoNiAlSc $(12 x+S c)$ and TiZrHfNbVCrMoMnFeCoNiAlBe $(12 x+B e)$ HEAs. Space group $\mathrm{P}_{3} / \mathrm{mmc}$.

\begin{tabular}{lcccc}
\hline parameter & $7 \mathrm{x}$ & $12 \mathrm{x}$ & $12 \mathrm{x}+\mathrm{Sc}$ & $12 \mathrm{x}+\mathrm{Be}$ \\
\hline $\mathrm{a}, \AA$ & $5.1923(3)$ & $5.03234(6)$ & $5.07770(2)$ & $4.9835(9)$ \\
$\mathrm{c}, \AA$ & $8.436(1)$ & $8.2041(2)$ & $8.2778(5)$ & $8.1298(4)$ \\
$\mathrm{c} / \mathrm{a}$ & 1.625 & 1.630 & 1.630 & 1.631 \\
$\mathrm{~V}, \AA^{3}$ & $196.96(5)$ & $179.930(4)$ & $184.78(1)$ & $174.859(9)$ \\
$R_{P}(\%)$ & 6.7 & 3.3 & 10.3 & 3 \\
$R_{W P}(\%)$ & 8.5 & 4.4 & 14.0 & 5 \\
$R_{(}\left(F^{2}\right)(\%)$ & 7.4 & 6.7 & 8.4 & 7 \\
$\chi^{2}(\%)$ & 2.5 & 1.3 & 4.5 & 1.01 \\
$\varepsilon \times 10^{-3}$ & 2.6 & 2.6 & 1.9 & 3.9 \\
\hline
\end{tabular}

fractions of these phases are small and the peaks of the main C14 phase are broad due to strong disorder. We suggest that $7 x$ alloy contain three phases because two additional peaks are hardly related to the same structure. Additional conclusions regarding the structure of these impurity phases can be made using electronic microscopy data (see discussion below).

We have performed the calculation of the unit cell parameters for C14 phases by the Rietveld method using the starting model [23] (see Tab. 1). It can be seen from the Tab. 1 that, in all the alloys studied, the ratios of the unit cell parameters $\mathrm{c} / \mathrm{a}$ are close to the ideal value of 1.633 for a close-packed hexagonal lattice (HCP). A slight compression of the unit cells during annealing may indicate a certain redistribution of elements over nonequivalent crystallographic positions.

We also perform an analysis of the integral width of the diffraction lines by the Williamson-Hall method to determine some microstructure parameters. These results indicate that the broadening of the diffraction lines is caused only by microstrains. The estimated microstrain values are presented in Tab. 1 . We see that the deformation degree $(\varepsilon)$ is rather high for all systems, especially for $12 x+B e$. The most probable reason for the stressed state in the HELPs is the occupation of crystallographic positions by atoms of various types (having different radii and different electronic structures).

\subsection{Optical and SEM analysis}

The microstructure of the Laves phases has been characterized with both optical and electron microscopy methods. The optical images allow revealing the microstructural morphology of the samples, while the electron microscopy imaging gives mainly details for chemical elements distribution. As seen in Fig. 2a, the 7x sample demonstrates a fine dendritic microstructure. The BSD scanning made over the whole surface of the intermetallic compound does not reveal significant variations in chemical composition or obvious signs for impurity phases. We find that dendrites and interdendritic regions have practically the same chemical compositions. The results of local analysis for the specific regions indicated by arrows in Fig. 2 b are collected in Tab. 2. Scanning over a large sample surface $(100 \times 100 \mu \mathrm{m})$ reveals that the weighted overall chemical composition in $7 x$ alloy is very similar to the equiatomic ratio. The chemical inhomogeneity detected by BSD-SEM is likely due to either the dendritic crystallization process or the precipitation of additional phases. The as-cast alloy demonstrates much more pronounced chemical inhomogeneity compared with the annealed sample. During the thermal processing, the compound partially relaxes but retains its initial dendritic structure. It is obvious that more prolonged exposition time is required to completely homogenize the crystalline structure in this refractory intermetallic.

Microscopy scanning performed on duodenary $12 x$ alloy has revealed its dendritic microstructure and rather homogeneous distribution of the constituent metals over the dendritic matrix. We find no detectable changes in the HEA microstructure during thermal processing; the alloy completely retains its initial dendritic morphology after 


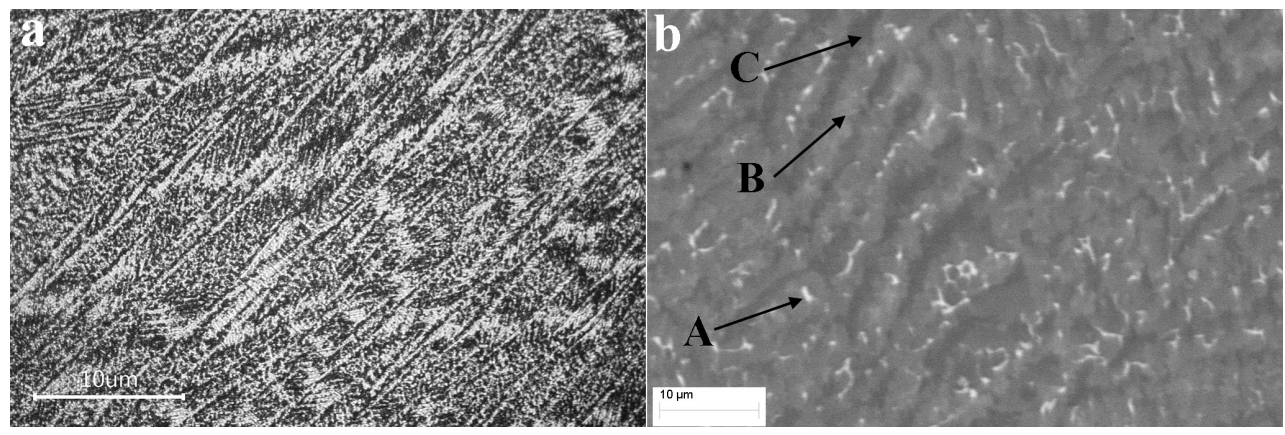

Figure 2. Optical micrograph (a) and backscattered electron SEM images (b) for the 7x sample. Arrows indicate the specific regions with different chemical element contrast.

Table 2: Chemical element distribution in TiZrHfNbCoNiAl (7x) high entropy Laves phase. The regions named as $\mathrm{A}, \mathrm{B}$, and $\mathrm{C}$ refer to the corresponding microareas illustrated in Fig. 2b.

\begin{tabular}{lccccccc}
\hline region & $\mathrm{Ti}$ & $\mathrm{Zr}$ & $\mathrm{Hf}$ & $\mathrm{Nb}$ & $\mathrm{Co}$ & $\mathrm{Ni}$ & $\mathrm{Al}$ \\
\hline $\mathrm{A}$ & 13.93 & 16.44 & 15.70 & 14.93 & 14.08 & 14.36 & 10.56 \\
$\mathrm{~B}$ & 16.28 & 13.44 & 14.75 & 12.23 & 14.11 & 14.30 & 14.89 \\
$\mathrm{C}$ & 12.73 & 14.10 & 13.87 & 17.56 & 15.78 & 11.80 & 14.16 \\
\hline
\end{tabular}

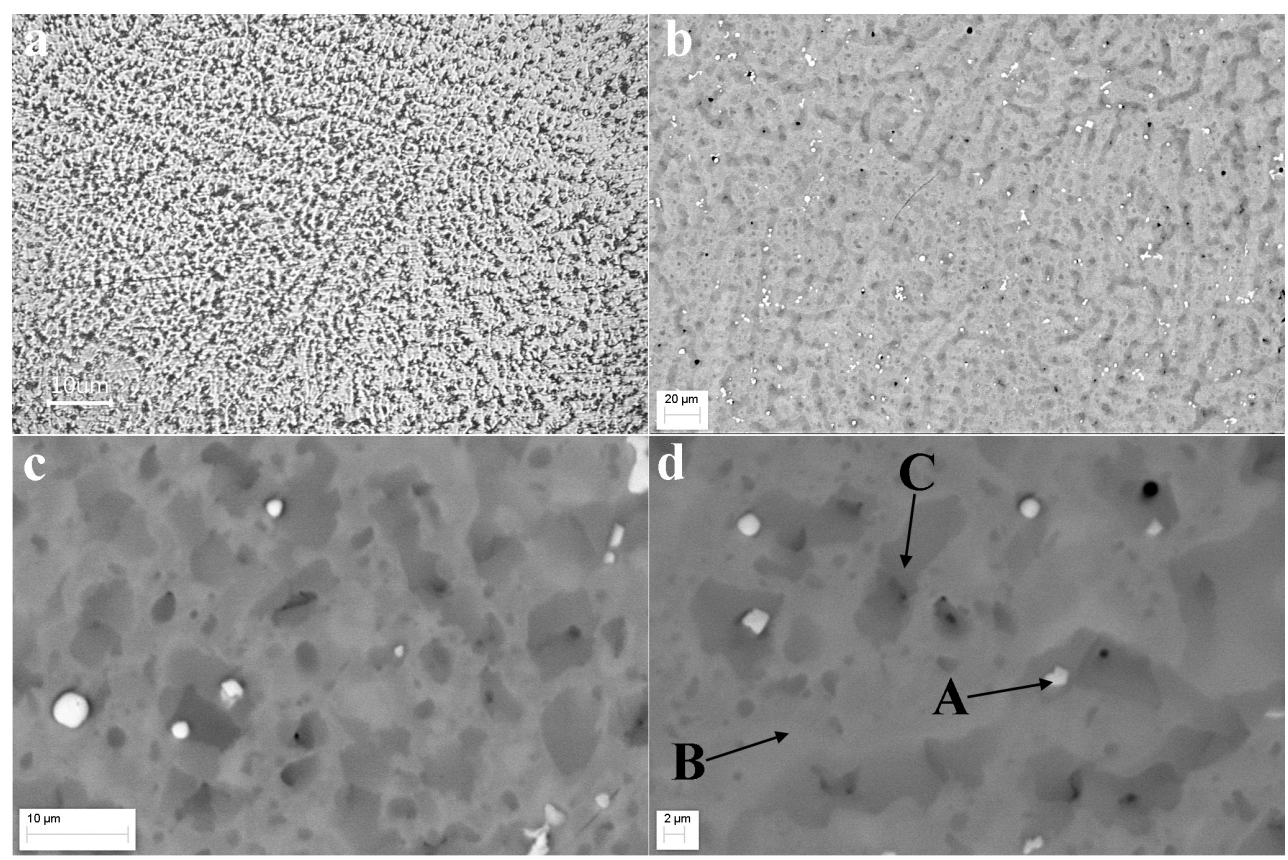

Figure 3. Optical micrograph (a) and backscattered electron SEM images $(b, c, d)$ for the $12 x$ sample. Arrows indicate the specific regions with different chemical element contrast. 
Table 3: Chemical element distribution in TiZrHfNbVCrMoMnFeCoNiAl (12x) high entropy Laves phase.

\begin{tabular}{lccc}
\hline element & white (A) & gray (B) & dark (C) \\
\hline $\mathrm{Ti}$ & 5.35 & 6.94 & 11.65 \\
$\mathrm{Zr}$ & 19.87 & 8.94 & 8.65 \\
$\mathrm{Hf}$ & 46.37 & 9.22 & 6.84 \\
$\mathrm{Nb}$ & - & 9.89 & 8.80 \\
$\mathrm{~V}$ & 3.79 & 8.59 & 8.46 \\
$\mathrm{Cr}$ & 3.55 & 9.19 & 7.10 \\
$\mathrm{Mo}$ & - & 9.46 & 8.04 \\
$\mathrm{Mn}$ & 2.28 & 5.06 & 4.66 \\
$\mathrm{Fe}$ & 4.27 & 9.19 & 7.91 \\
$\mathrm{Co}$ & 4.15 & 8.02 & 8.86 \\
$\mathrm{Ni}$ & 5.14 & 7.36 & 10.41 \\
$\mathrm{Al}$ & 5.26 & 8.15 & 8.61 \\
\hline
\end{tabular}

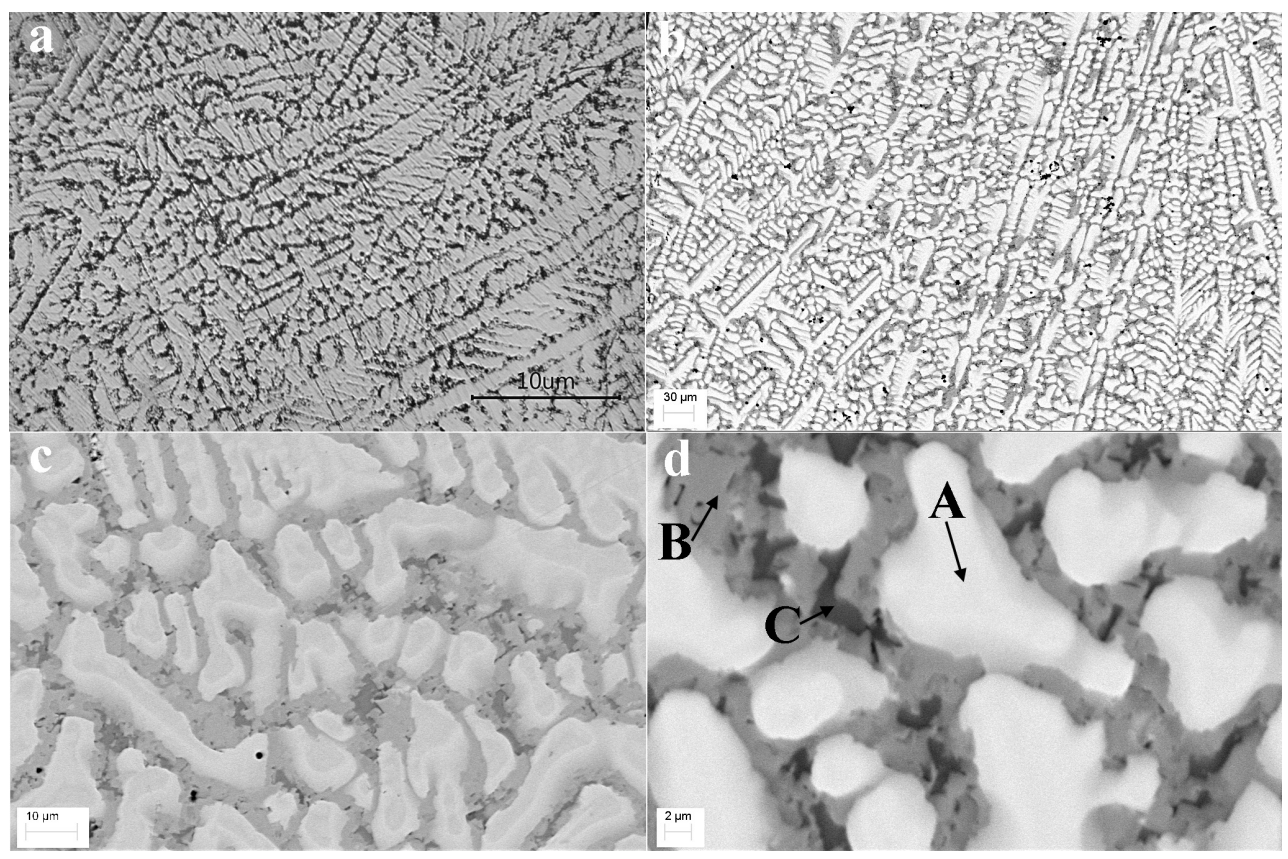

Figure 4. Optical micrograph (a) and backscattered electron SEM images $(b, c, d)$ for the $12 x-S c$ sample. Arrows indicate the specific regions with different chemical element contrast.

annealing. The chemical composition of the dendrites well corresponds to the nominal element ratio. The interdendritic areas (gray zones in Fig. 3d) are enriched in $\mathrm{Ti}$ and $\mathrm{Ni}$, see the results of the local analysis in Tab. 3. Interesting sphere-like precipitations of 1-2 $\mu \mathrm{m}$ in diameter are observed along the dendritic boundaries, see white inclusions in Fig. 3b-d. These precipitations are distributed evenly over the alloy matrix. Their chemical composition was found to deviate significantly from the dendritic structures. We have detected with EDX that hafnium and zirconium predominate in the chemical composition of these inclusions. Note that the precipitations take place for both the as-cast and the thermally treated samples. We see no noticeable changes in the structural morphology of the dendrites and the spherical inclusions after annealing. And chemical element distribution over the alloy microstructure retains practically the same. The microstructure inhomogeneities revealed with the microscopy tests confirm the fact that the refractory HEA needs high temperatures and very long exposure times to be homogenized. Note that the HEAs under consideration have large values of the configurational entropy, $\Delta S_{\text {conf }}$ is $1.95-2.56 R$. All that drastically slows down the thermal relaxation processes.

As follows from the microscopy observations performed on the alloy $12 x+S c$, both the as-cast and heat-treated samples demonstrate a fine dendritic microstructure, see the optic micrograph in Fig. 4a. Backscattered electron SEM images (Fig. 4b-d) reveal 
Table 4: Chemical element distribution in TiZrHfNbVCrMoMnFeCoNiAlSc $(12 \mathrm{x}+\mathrm{Sc})$ high entropy Laves phase.

\begin{tabular}{lccc}
\hline element & white (A) & gray (B) & dark (C) \\
\hline $\mathrm{Ti}$ & 4.79 & 12.20 & 6.26 \\
$\mathrm{Zr}$ & 7.91 & 8.59 & 5.26 \\
$\mathrm{Hf}$ & 10.47 & 5.59 & 3.82 \\
$\mathrm{Nb}$ & 10.21 & 7.34 & 1.72 \\
$\mathrm{~V}$ & 8.39 & 6.33 & 1.09 \\
$\mathrm{Cr}$ & 11.73 & 4.56 & 0.92 \\
$\mathrm{Mo}$ & 9.76 & 4.41 & 0.69 \\
$\mathrm{Mn}$ & 6.74 & 5.87 & 1.04 \\
$\mathrm{Fe}$ & 9.74 & 6.57 & 1.45 \\
$\mathrm{Co}$ & 6.51 & 10.25 & 7.27 \\
$\mathrm{Ni}$ & 2.73 & 10.51 & 34.68 \\
$\mathrm{Al}$ & 7.52 & 8.44 & 3.92 \\
$\mathrm{Sc}$ & 3.52 & 9.34 & 31.87 \\
\hline
\end{tabular}

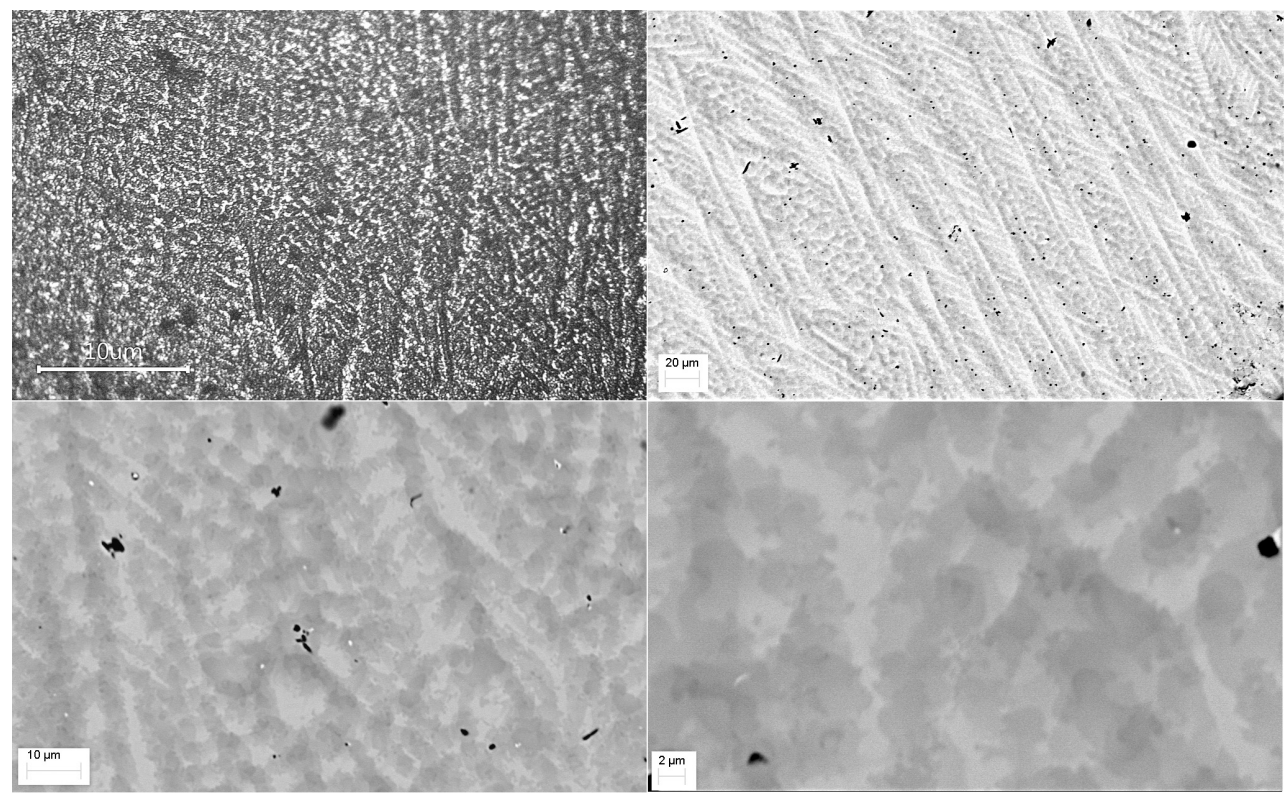

Figure 5. Optical micrograph (a) and backscattered electron SEM images (b,c,d) for the 12x-Be sample.

clear chemical element segregation between dendrites and interdendritic regions. At least three specific microareas can be recognized, as indicated by arrows in Fig. $4 \mathrm{~d}$. The results of local chemical analysis for these regions are given in Tab. 4. According to EDX analysis, the dendritic grains are enriched in refractory elements ( $\mathrm{Nf}, \mathrm{Nb}, \mathrm{Mo}$, and $\mathrm{Cr}$ ), while the grain boundaries (grey fields) contain an excess of $\mathrm{Ti}, \mathrm{Ni}$, and $\mathrm{Co}$. Significant chemical inhomogeneity is observed for interdendritic regions, where scandium and nickel elements dominate. One can conclude that scandium due to its large atomic radius tends to segregate from the parent phase and form an additional one. These dark precipitations enriched in Sc and Ni metals can be classified as inclusions of a secondary phase. The microscopic observations confirm dual-phase microstructure for the HEA $12 x+$ Sc detected with XRD analysis. Note that microstructure morphology and element redistribution in the alloy before and after thermal treating are practically the same. This fact indicates that the HEA is a thermally stable dual-phase material.

The HEA containing beryllium demonstrates different microstructure morphology compared with other multicomponent alloys investigated. As seen in Fig. 5, the sample has an ultra-fine dendritic microstructure with an almost homogenous distribution of the constituted elements over the alloy matrix. Local variations in chemical composition are within the EDX sensor resolution. According to XRD results, $12 x+$ Be alloy is a single-phase $\mathrm{C} 14$ compound with very strong structural defectiveness compared with 
other explored HEAs. The SEM data confirm completely this result and so we report the formation of a new stable single-phase HELP.

Summarizing the data obtained by XRD and SEM methods, we can make additional conclusions regarding the structure of the systems under consideration. First, according to $\mathrm{XRD}, 7 \mathrm{x}$ alloy contains the main $\mathrm{C} 14$ Laves phase and impurity phases which are detectable by only two low-intensity peaks. The SEM results confirm the existence of three phases and give us their compositions which are close to the equiatomic one. Of course, unequivocal determination of a structure by one XRD peak is impossible. However, we can suggest the candidate structures by using phase compositions obtained by BSD-SEM analysis. Doing so we conclude that the most probable structures for impurity phases in 7x alloys are C14 $(a=4.9 \AA, c=5.93 \AA)$ and C15 $(a=11.3 \AA)$ Laves phases. Such a situation when the system is a mixture of different Laves phase polytypes is common enough [24].

Similar analysis can be performed for $12 x+S c$ alloy. XRD analysis reveals one lowintensity peak which cannot be assigned to $\mathrm{C} 14$ Laves phase. SEM analysis confirms the existence of the second phase, which is almost evenly enriched in Ni and Sc. Taking this information into account, we suggest that the undetectable XRD peak can be assigned to a solid solution based on either NiSc compound (space group Pm-3m) or $\mathrm{Ni}_{2} \mathrm{Sc}$ one (C15 Laves phase). The latter variant seems more favorable due to general tendency to form multi-Laves-phase structures.

The most intriguing situation takes place for the $12 x$ system. XRD analysis detects no phases but C14 Laves intermetallic. This result is carefully confirmed by Rietveld refinement; all the detected peaks have been perfectly described by the single-phase C14 structure model (see also Ref. [18]). However, SEM analysis reveals sphere-like precipitations along the dendritic boundaries (see white inclusions in Fig. 3b-d) whose chemical composition is strongly enriched in hafnium and zirconium. To explain this fact, we can make two hypotheses: (i) 12x alloy is a single-phase HELP with a complicated microstructure and strongly inhomogeneous element distribution due to dendritic segregation effects; (ii) the alloy is a mixture of a dominant C14 phase and an impurity phase whose XRD peaks overlap the peaks of the main phase. It should be noticed the composition of these precipitations obeys well the general formula $A_{2} B$, where $\mathrm{A}=(\mathrm{Zr}$, $\mathrm{Hf})$, and $\mathrm{B}$ - other elements. Taking into account that $\mathrm{Hf}$ and $\mathrm{Zr}$ have the largest atomic sizes among the elements containing a $12 x$ system, we can guess that we deal with the second phase which is a solid solution based on $\mathrm{Hf}_{2} \mathrm{Co} \mathrm{C} 15$ Laves phase. We check that the most intensive reflection (511) overlaps the peak (103) of the main C14 phase, and therefore the $\mathrm{Hf}_{2} \mathrm{Co}$ phase is not detected by XRD).

\subsection{Specific heat}

The complicated chemical composition and defective crystalline structure of the samples allow one to expect extraordinary physical properties. To characterize HELPs under consideration, we have measured their specific heat capacity, see Fig.6. These measurements serve the twofold purpose of revealing abnormal specific heat contribution and determining the Debye characteristic temperature. We see that all the intermetallics demonstrate typical behavior of the property with temperature.

The specific heat of the samples near the room temperatures is about that predicted by the Dulong-Petit law. At temperatures above $300 \mathrm{~K}$, the $\mathrm{C}(\mathrm{T})$ dependencies are linear with a weak positive slope. These observations motivate us to conclude that there are no abnormal specific heat contributions. We suggest that prevailing standard lattice contribution and an additional linear term determine the specific heat capacity of the intermetallics. The linear increase in the specific heat with temperature is due to both the conduction electrons and anharmonic lattice vibrations. Formally, these linear 


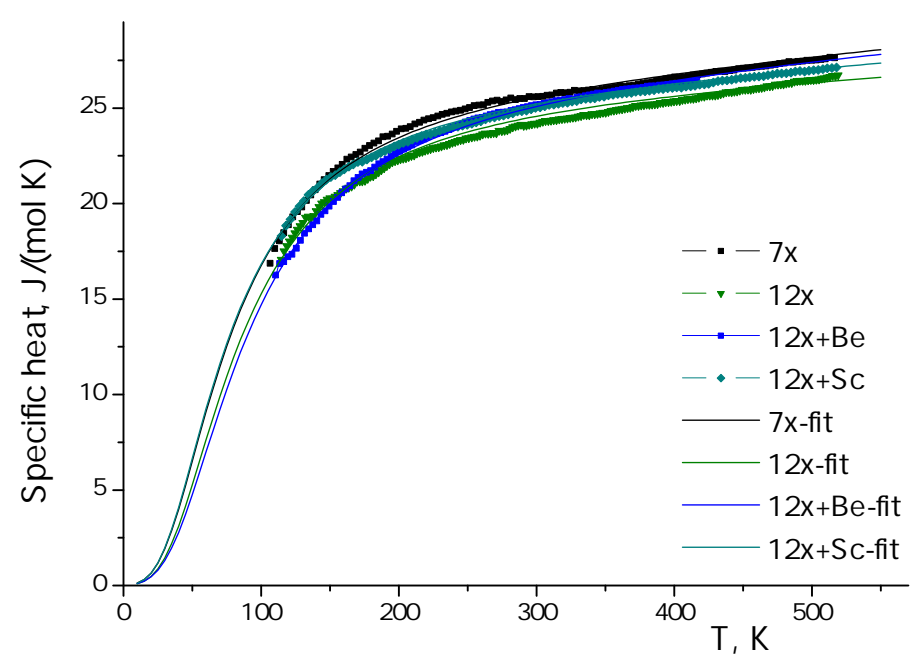

Figure 6. Specific heat for the annealed samples of TiZrHfNbCoNiAl (7x), TiZrHfNbVCrMoMnFeCoNiAl (12x), TiZrHfNbVCrMoMnFeCoNiAlSc $(12 x+S c)$ and TiZrHfNbVCrMoMnFeCoNiAlBe $(12 x+\mathrm{Be})$ HEAs. Dots represent the experimental data; solid lines are the best fitting to the equation 1.

contributions can be taken into account as $\beta T$, where $\beta$ is the temperature coefficient. We modify the standard Debye model to fit the experimental data in the following form:

$$
C(T)=9 R\left(\frac{T}{\Theta_{D}}\right)^{3} \int_{0}^{\Theta_{D} / T} \frac{e^{x} x^{4}}{\left(e^{x}-1\right)^{2}} d x+\beta T
$$

where $\mathrm{R}$ is the molar gas constant, $\Theta_{D}$ is the Debye characteristic temperature, the second term is the specific heat contribution due to the conduction electrons and anharmonic lattice vibrations. It is worth noting that the equation 1 describes the isochoric specific heat $C_{V}$, while we measure isobaric one $C_{P}$. We neglect thermal expansion effects and accept that $C_{V} \cong C_{P}$. As is well known, in the case of solids this approach is completely adequate. The best-fitting coefficients for Eq.1 are collected in Tab.5. One can see that the Debye temperatures of the HEAs are practically the same. It seems reasonable that the alloys with an identical crystalline structure and similar chemical compositions demonstrate analogous vibrational properties. The temperature coefficient $\beta$ for the alloys is too small to discuss extensively. This only means that both electron and anharmonic contributions in the specific heat of the Laves phases are not anomalous. Summarising, we conclude that the chemical complexity and strongly defected crystalline structure of the fabricated alloys do not provide enhanced specific heat or its abnormal behavior with temperature.

\subsection{Electrical resistivity}

All the fabricated Laves phases demonstrate high values of the resistance expected for intermetallic compounds of this type, see Fig.7. The $7 x$ and $12 x$ samples reveal a pronounced Kondo-like anomaly below which the resistivity noticeably increases. This feature is absent for the HEAs containing scandium and beryllium. The $\rho(T)$ curves of the samples above $100-150 \mathrm{~K}$ have a positive slope that indicates metallic conductivity for all the phases. We have measured the resistivity on both the as-cast and annealed samples and found no qualitative changes in the resistance behavior after the thermal treatment. As expected for a thermally relaxed crystal structure, the homogenized alloys show reduced electrical resistivity.

The observed temperature behavior of the resistance is obviously the result of a superposition of several factors. Indeed, we deal with the HEAs that have strongly 


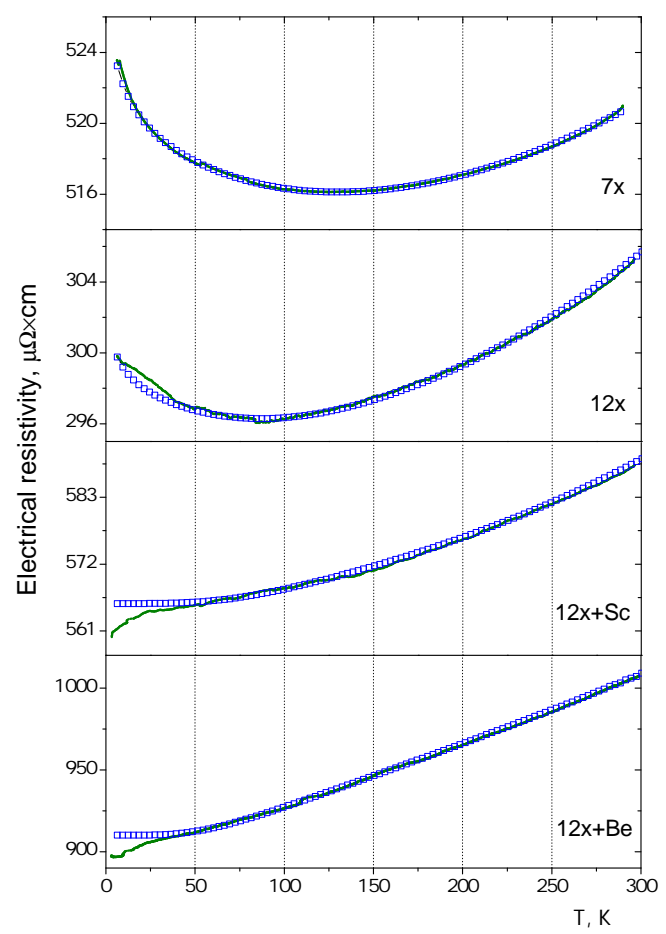

Figure 7. Electrical resistivity of the Laves phases vs. temperature. Solid lines are experimental data; the dots are the best fitting to the equation 2 . The sample names given in the legend refer to the compound compositions listed in Tab.5.

distorted crystal lattices comprising different magnetic and non-magnetic transition elements redistributed over crystallographic positions in a stochastic way. Thus, we have to account for different scattering mechanisms to describe correctly the experimental results. A modified Bloch-Grüneisen equation is utilized for the fitting:

$$
\rho(T)=\rho_{0}+\frac{A}{\Theta_{D}} \rho_{\mathrm{el}-\mathrm{ph}}(T)+C T^{3}+D \ln \frac{\mu}{T}
$$

where $\rho_{0}$ is the residual resistivity due to static lattice defects and uncontrolled impurities, the second term describes electron-phonon scattering, $\Theta_{D}$ is Debye characteristic temperature, the third term is the resistance contribution due to Mott's $s-d$ interband scattering, and the last one is the Kondo contribution term. Note that this equation includes many adjusting parameters that complicate the fitting procedure and, therefore, there is a risk of misinterpretation of the results. To simplify the approximation, we accept the Debye temperatures for each composition equal to those determined from the specific heat measurements, see Tab.5. When the Debye parameter is fixed, the approximation goes correctly. So we extract the following electronic parameters given in Tab.5. One can see that the $\rho_{0}$ component is the main contribution to the HEA resistance. Such values are expectable for materials with a defected crystalline structure and ultra-fine microstructure. Another interesting fact is that the term describing Mott's $s-d$ interband scattering is rather large, which indicates a significant role of $d$-electrons in the electrical conductivity of the HEA. This situation is very expectable because the considered Laves phase consists predominantly of $d$-transition metals. For the samples $7 \mathrm{x}$ and $12 \mathrm{x}$, the Kondo temperatures $\mu$ estimated during the fitting procedure correlate well with the resistivity minimum positions in the $\rho(T)$ dependencies. This fact suggests that the performed data approximation is correct. Based on the resistance results, we conclude that the defected crystalline structure and chemical complexity inherent to the fabricated alloys are the main reasons for the high electrical resistivity of the high entropy Laves phases and its complicated temperature behavior. 
Table 5: Parameters of the equations 1 and 2 providing the best fit of the experimental specific heat and resistivity for TiZrHfNbCoNiAl (7x), TiZrHfNbVCrMoMnFeCoNiAl (12x), TiZrHfNbVCrMoMnFeCoNiAlSc $(12 x+S c)$ and TiZrHfNbVCrMoMnFeCoNiAlBe $(12 x+B e)$ high entropy Laves phases.

\begin{tabular}{|c|c|c|c|c|c|c|c|}
\hline sample & $\rho_{0}, \mu \mathbf{\square} \times \mathrm{cm}$ & $\Theta_{D}, K$ & $\beta, \mathrm{mJ} /\left(\mathrm{mol} \times K^{2}\right)$ & $A, \mu \boxminus \times \mathrm{cm} \times K$ & $C, \mu \mathrm{m} \times \mathrm{cm} \times K^{-3}$ & $D, \mu \mathbf{m} \times \mathrm{cm}$ & $\mu, K$ \\
\hline $7 x$ & 515.4 & 310 & 64 & $0.12 \times 10^{-4}$ & $2.85 \times 10^{-15}$ & $255 \times 10^{-10}$ & 120 \\
\hline $12 x$ & 295.4 & 340 & 39 & $1.02 \times 10^{-4}$ & $2.45 \times 10^{-15}$ & $195 \times 10^{-10}$ & 80 \\
\hline $12 x+S c$ & 565.1 & 305 & 51 & $1.55 \times 10^{-4}$ & $4.53 \times 10^{-15}$ & - & - \\
\hline $12 x+B e$ & 910.0 & 360 & 62 & $15.85 \times 10^{-4}$ & $4.85 \times 10^{-15}$ & - & - \\
\hline
\end{tabular}

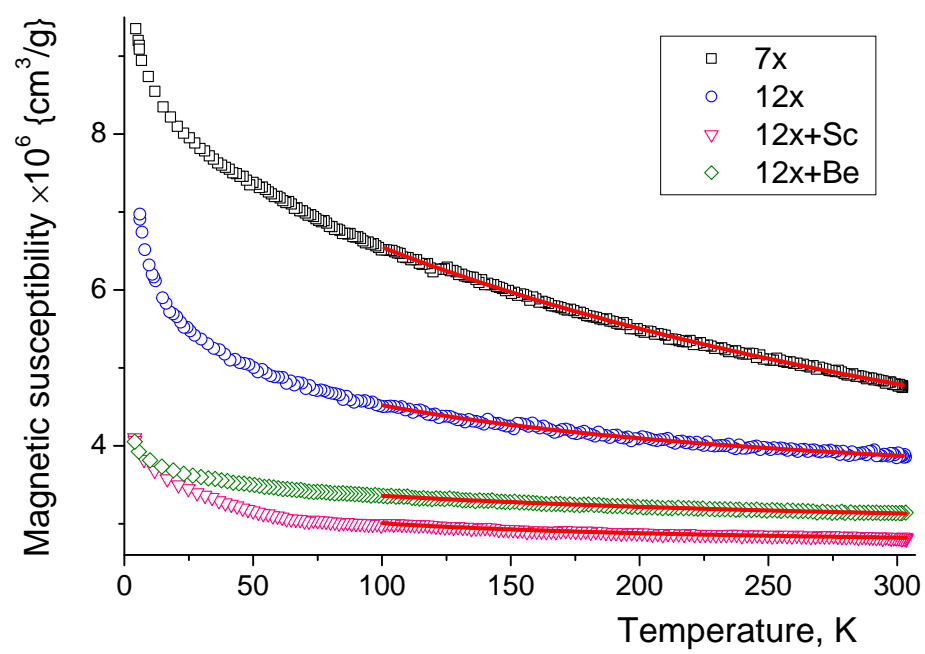

Figure 8. Magnetic susceptibility of the Laves phases vs. temperature. Dots are experimental data; the solid lines are the best fitting to the Curie-Weiss law. The sample names given in the legend refer to the compound compositions listed in Tab.5.

\subsection{Magnetic properties}

The alloys under consideration are mixtures of various transitions elements, which allows one to expect unusual magnetism. We have measured the mass magnetic susceptibility for the HEAs in the range of $4-300 \mathrm{~K}$, see Fig. 8. All the phases demonstrate weak paramagnetic susceptibility over the explored temperature interval. No signs for magnetic ordering was found. We tested both as-cast and annealed samples of the alloys to prove possible evolution in magnetic properties during the thermal processing [18]. No significant changes were found in magnetism between the samples measured, indicating the thermal stability of the Laves phase. Analysis of the magnetic data reveal that the heat-treated samples are paramagnets whose magnetic susceptibility can be successfully described by the generalized Curie-Weiss law $\chi=\chi_{0}+C /(T-\Theta)$ in the interval of $300 \mathrm{~K}-100 \mathrm{~K}$. At temperatures below $100 \mathrm{~K}$, the curve fitting is not well due to long-range and short-range magnetic correlations between uncompensated magnetic moments or the Kondo anomaly.

The experimental data have been fitted with the parameters listed in Table. 6.

Table 6: Magnetic characteristics of the HEAs: temperature-independent magnetic susceptibility contribution $\chi_{0}$, paramagnetic Curie temperature $\Theta$, effective magnetic

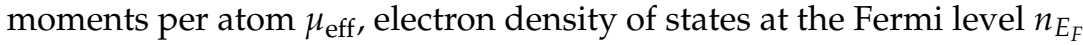

\begin{tabular}{lcccc}
\hline sample & $\chi_{0}\left(\mathrm{~cm}^{3} / g\right)$ & $\Theta(\mathrm{K})$ & $\mu_{\mathrm{eff}}\left(\mu_{B}\right)$ & $n_{E_{F}}\left(\mathrm{eV}^{-1}\right)$ \\
\hline $7 \mathrm{x}$ & $1.02 \times 10^{-6}$ & -329 & 1.23 & 0.8 \\
$12 \mathrm{x}$ & $3.13 \times 10^{-6}$ & -130 & 0.43 & 2.3 \\
$12 \mathrm{x}+\mathrm{Sc}$ & $2.67 \times 10^{-6}$ & -60 & 0.17 & 1.9 \\
$12 \mathrm{x}+\mathrm{Be}$ & $2.80 \times 10^{-6}$ & -195 & 0.29 & 1.9 \\
\hline
\end{tabular}



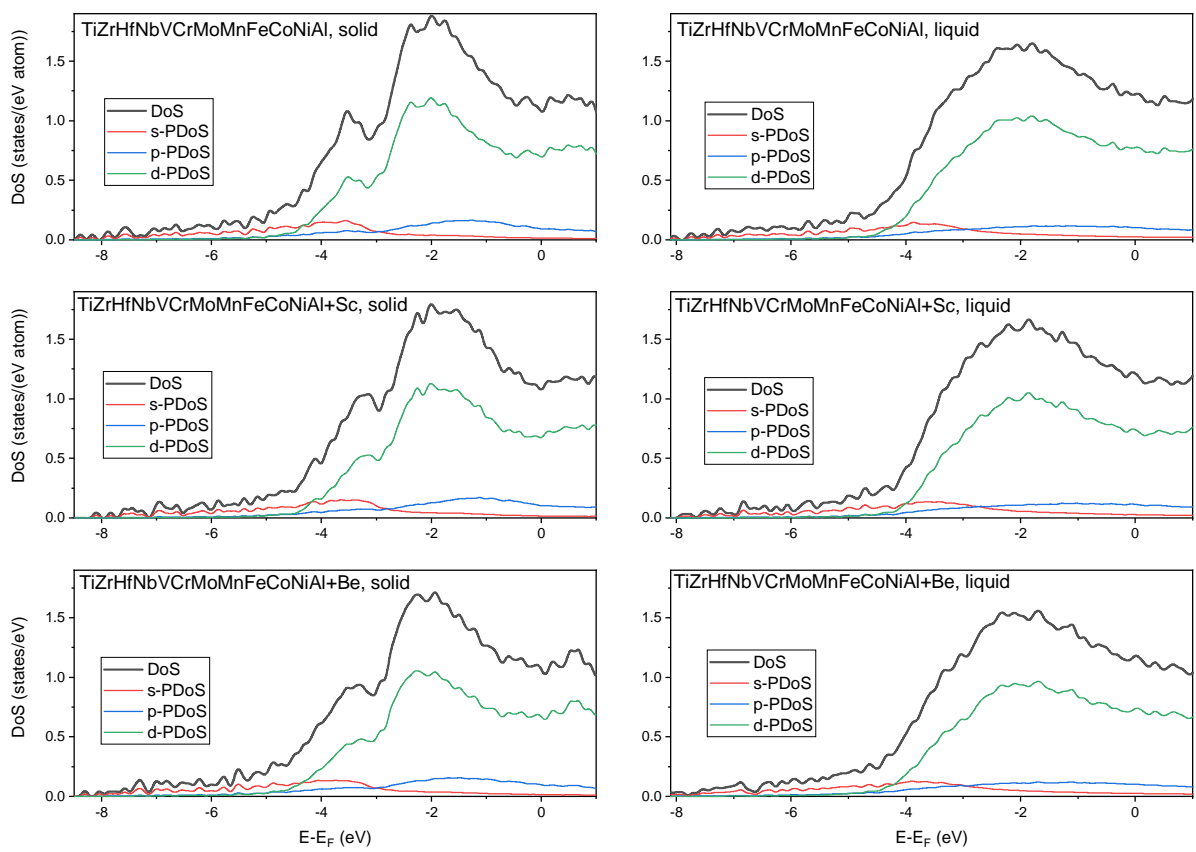

Figure 9. Electronic densities of states for HELPs $12 x, 12 x+S c, 12 x+B$ e calculated for $3 \times 3 \times 3$ supercells of $\mathrm{C} 14$ Laves phase with random distributions of the elements on the lattice cites (left panels) and for 512-atom supercell of liquid configuration (right panels).

One can see that the paramagnetic temperatures $\Theta_{p}$ for the HEA samples are large and negative that indicates strong antiferromagnetic correlations. The effective magnetic moments calculated for the HEAs are noticeably less than those expected from an additive mixture of neutral atoms. So we can conclude that a part of $d$-electrons that determine magnetism of the system is involved in chemical bonding. From the temperature-independent susceptibility contribution $\chi_{0}$, we estimate the value of the electron density of states (DOS) at the Fermi level. Briefly, we extract the contribution of Pauli susceptibility $\chi_{P}$ into $\chi_{0}$ and then apply the formula $\chi_{P}=2 S \mu_{B}^{2} n\left(E_{F}\right)$, where $S$ is Stoner exchange parameter, which reflects effects of electronic correlations [25] (we set $S=1.5$ ). This estimation gives rather high values for $n\left(E_{F}\right)$ that suggests that the $d$-orbitals dominate the conduction band of the compounds. The existence of the $s-d$ hybridization gives an essential contribution to the electron scattering processes, providing high electrical resistivity of the HEAs. On the other hand, the enhanced DOS means that no energy gap or pseudogap is formed in the intermetallics. The experimentally discovered metallic type of conductivity in the phases also confirms this fact. Summarising, we can conclude that the explored multicomponent Laves phases are ordinary weak paramagnets of Curie-Weiss-like type down to liquid helium temperatures.

\subsection{Ab initio calculations}

To address the electronic structure and properties of interatomic interaction in the system, we perform $a b$ initio calculations utilizing density functional theory as implemented in VASP code [26]. Projector augmented-wave (PAW) pseudopotentials and Perdew-Burke-Ernzerhof (PBE) $[27,28]$ gradient approximation to the exchangecorrelation functional were applied [29]. The wave function was expanded by the plane wave basis set in the periodic boundary condition, and plane wave cut-off energy was $500 \mathrm{eV}$. Only $\Gamma$-point was used for sampling Brillouin zone because rather large supercells were considered (see below).

The calculation of the electronic structure of disordered multicomponent solid solutions is a non-trivial task. For the 2-4 component systems, the special quasirandom 
structures (SQSs) are usually utilized for that purpose [30,31]. Since, generating SQSs for 12-13 component systems in a usual way is a difficult task, we apply two alternative ways. First, we calculate DOSs for $3 \times 3 \times 3$ supercells of the $\mathrm{C} 14$ Laves phase ( 327 atoms) and permutate the alloy components on the lattice using an evolutionary algorithm as implemented in the USPEX code [32]. The algorithm searches for a configuration that maximizes the configurational disorder described via a properly designed structural fingerprint and gives a generalized version of the SQS [33]. Second, we consider supercells of 512 atoms at thermodynamic states corresponding to the liquid phase. Equilibrium configurations were generated via ab initio molecular dynamics using the protocol described in [34].

The resulted DOSs are presented in Fig. 9. We see that the general properties of DOSs for liquid and solid states are qualitatively the same that suggests strong structural disorder in the systems under consideration. Total DOSs for all three systems demonstrate purely metallic behavior with a rather high values near the Fermi level. The projected DOSs expectedly reveal a dominant contribution of $d$-states in total DOSs. Note that DOSs calculated in both solid and liquid states and for all systems reveal the same value on the Fermi level $n\left(E_{F}\right) \approx 1.2 \mathrm{eV}^{-1}$, which is the same order as evaluated from experimental data on the magnetic susceptibility. The difference between these values is caused by the fact that the formula related $n\left(E_{F}\right)$ and Pauli susceptibility is obtained from the free electron model. The Stoner parameter applied to take into account the electron correlations can not be obtained explicitly and thus the $n\left(E_{F}\right)$ extracted from experimental data is only the estimation of the order of magnitude. The fact that electronic structure of all the alloys is practically the same supports the idea that experimentally observed difference in the behavior of the resistivity in HELPs under study is mostly caused by the difference in their microstructure.

\section{Discussion and conclusions}

We have addressed Laves phase formation in multicomponent multi-principal element metallic alloys as well as their structural, thermodynamic, electronic, and magnetic properties. We consider duodenary TiZrHfNbVCrMoMnFeCoNiAl (12x) alloy, in which the formation of single-phase C14 Laves phase has been recently reported [18], and study three alloys based on $12 x$ : $7 x=12 x-\mathrm{VCrMoMnFe}, 12 \mathrm{x}+\mathrm{Sc}, 12 \mathrm{x}+\mathrm{Be}$. We observe that all three systems crystalize with the formation of $\mathrm{C} 14$ Laves phase as a dominant structure. However, the microstructure and phase composition of the alloys under consideration are essentially different. The alloy with the addition of beryllium $12 x+B e$ reveals a single-phase $\mathrm{C} 14$ structure with a very high concentration of structural defects and ultra-fine dendritic microstructure with an almost homogenous distribution of the constituted elements over the alloy matrix. The $7 x$ alloy is a mixture of three phases: the main C14 Laves phase and two impurity phases, which are probably also Laves phases with C14 and C15 structures and slightly different compositions. The $12 x+S c$ alloy is a two-phase material with $\mathrm{C} 14$ as the main phase and NiSc-based solid solution as an impurity one. Note that all the fabricated structures demonstrate thermal stability; we did not see any noticeable structural changes after isothermal annealing at $\mathrm{T}=673 \mathrm{~K}$ for $50 \mathrm{~h}$.

From the point of view of high-entropy intermetallic development, the most interesting result is fabricating a new single-phase HELP in $12 x+B e$ alloy. We can conclude that beryllium addition to the $12 x$ composition facilitates the formation of a chemically homogenous single-phase solid solution. The remarkable level of structural defects observed in this alloy can be explained by the small atomic size of the beryllium. This metal can easily replace and occupy crystallographic positions in lattice sites, forming a substitution solid solution. Besides, an interstitial solid solution can be formed because small beryllium atoms can also occupy the interstitial lattice positions. In the case of the system under consideration, both solid solution types can be formed simultaneously, providing strong structural distortions. 
From a practical viewpoint, the explored high entropy intermetallics do not demonstrate extraordinary physical properties. Complex chemical composition and defective crystalline structure of the phases have a significant impact on the electrical resistivity only. All the HELPs are high-resistivity but metallic materials. Other explored physical properties behave as usual. So we find typical for many solids temperature dependencies of the specific heat obeying classical Debye model. There are no abnormal specific heat contributions or exothermic/endothermic effects. The coexistence of various transition elements in the HELP lattice does not provide unusual magnetism or magnetic ordering. The explored HELPs are ordinary weak paramagnets of Curie-Weiss type down to liquid helium temperatures. All the intermetallics are characterized by the enhanced DOS, indicating that no energy gaps or pseudogaps are formed in the systems.

Taking into account the above findings as well as previously reported results [12$16,16,17]$, we can conclude that the formation of Laves phases in multicomponent multiprincipal element metallic alloys is a surprisingly universal phenomenon. Indeed, considering multicomponent mixtures of metals with different atomic sizes and different electronic characteristics, we very often, and maybe even most often, observe solid solutions in which Laves phase (usually C14 one) is at least the dominant phase. The origin of this universality is not well understood. However, we argue that the mechanism of Laves phase stabilization is rather topological than electronic. The idea is when mixing many metals of differing atomic sizes, we get a system with a rather broad and continuous distribution of interparticle distances. From the point of view of solid phase formation, such systems behave effectively as polydisperse mixtures of hard (or soft) spheres. It is known, that such mixtures can form Laves phases at certain ranges of size dispersity [35]. This analogy between the structural formation in multicomponent metallic alloys and polydisperse hard-sphere mixtures is interesting and deserves separate investigation.

Another important and rather general conclusion is that multicomponent HELPs are not always single-phase materials but rather mixtures of several Laves phases. This behavior is a consequence of the fact that there are many Laves polytypes, which can compete during the solidification process [24]. This situation is well known in binary and ternary alloy and it is reasonable to expect that it is also a general feature of HELPforming multicomponent alloys. Even when a single-phase state is thermodynamically favorite, multi-Laves-phase HEAs may be actually stable due to very long relaxation times. Such a complex structure of HELPs makes it difficult to recognize all the phases in a system under investigation. Particularly, it is often not clear if the system is a true single-phase HELP even when XRD analysis and microscopy support this idea. When considering HELPs with different composition complexity, we find also that there is no clear correlation between configurational entropy and the Laves phases stabilization. The conclusion we draw from these findings is that configurational entropy is a negligible factor in the stabilization of HELPs.

Author Contributions: Uporov S.A.: Project administration, Funding acquisition, Writing - original draft; Ryltsev R.E.: Conceptualization, Formal analysis, Validation, Writing - review, editing; Estemirova S.Kh: Investigation, Writing - review, editing, Visualization; Sterkhov E.V.: Investigation, Formal analysis, Visualization; Cherepanova L.A.: Investigation, Formal analysis; Yagodin D.A.: Investigation, Formal analysis; Dubinin N.E.: Formal analysis, Validation; Chtchelkatchev N.M.: Methodology, Conceptualization, Formal analysis, Validation, Writing - review, editing.

Funding: This work was supported by Russian Science Foundation (RCF grant 19-73-20053). Ab initio calculations were performed within the frameworks of RCF grant 18-12-00438.

Data Availability Statement: The data that support the findings of this study are available on request from the corresponding author.

Acknowledgments: Experiments were performed using scientific instruments included in the Collective Equipment Center "Ural-M" and common Use Center of the Ural Branch of RAS "Geoanalyst". The numerical calculations are carried out using computing resources of the federal collective usage center 'Complex for Simulation and Data Processing for Mega-science Facilities' 
at NRC 'Kurchatov Institute' (ckp.nrcki.ru/) and Joint Supercomputer Center of Russian Academy of Sciences (www.jscc.ru).

Conflicts of Interest: The authors declare that they have no known competing financial interests or personal relationships that could have appeared to influence the work reported in this paper.
Abbreviations
The following abbreviations are used in this manuscript:

$\begin{array}{ll}\text { HEA } & \text { high-entropy alloys } \\ \text { HELP } & \text { high-entropy Laves phases } \\ \text { HEIC } & \text { high-entropy intermetallic compounds } \\ \text { SSS } & \text { simple solid solutions } \\ \text { VEC } & \text { valence electron concentration }\end{array}$

\section{References}

1. Biswas, K.; Yeh, J.W.; Bhattacharjee, P.P.; DeHosson, J.T. High entropy alloys: Key issues under passionate debate. Scripta Materialia 2020, 188, 54-58. doi:10.1016/j.scriptamat.2020.07.010.

2. George, E.P.; Raabe, D.; Ritchie, R.O. High-entropy alloys. Nature Reviews Materials 2019, 4, 515-534. doi:10.1038/s41578-0190121-4.

3. Miracle, D.; Senkov, O. A critical review of high entropy alloys and related concepts. Acta Materialia 2017, 122, 448-511. doi:https://doi.org/10.1016/j.actamat.2016.08.081.

4. Zhang, W.; Liaw, P.K.; Zhang, Y. Science and technology in high-entropy alloys. Science China Materials 2018, 61, 2-22. doi:10.1007/s40843-017-9195-8.

5. Praveen, S.; Kim, H.S. High-Entropy Alloys: Potential Candidates for High-Temperature Applications - An Overview. Advanced Engineering Materials 2018, 20, 1700645. doi:10.1002/adem.201700645.

6. $\quad$ Tsai, M.H.; Yeh, J.W. High-Entropy Alloys: A Critical Review. Materials Research Letters 2014, 2, 107-123. doi:10.1080/21663831.2014.912690.

7. Steurer, W. Single-phase high-entropy alloys - A critical update. Materials Characterization 2020, 162, 110179. doi:10.1016/j.matchar.2020.1101

8. Zhou, N.; Jiang, S.; Huang, T.; Qin, M.; Hu, T.; Luo, J. Single-phase high-entropy intermetallic compounds (HEICs): bridging high-entropy alloys and ceramics. Science Bulletin 2019, 64, 856-864. SPECIAL TOPIC: Electromagnetic Metasurfaces: from Concept to Applications, doi:10.1016/j.scib.2019.05.007.

9. Yao, K.; Liu, L.; Ren, J.; Guo, Y.; Liu, Y.; Cao, Y.; Feng, R.; Wu, F.; Qi, J.; Luo, J.; Liaw, P.K.; Chen, W. High-entropy intermetallic compound with ultra-high strength and thermal stability. Scripta Materialia 2021, 194, 113674. doi:10.1016/j.scriptamat.2020.113674.

10. Karati, A.; Nagini, M.; Ghosh, S.; Shabadi, R.; Pradeep, K.G.; Mallik, R.C.; Murty, B.S.; Varadaraju, U.V. Ti2NiCoSnSb - a new half-Heusler type high-entropy alloy showing simultaneous increase in Seebeck coefficient and electrical conductivity for thermoelectric applications. Scientific Reports 2019, 9, 5331. doi:10.1038/s41598-019-41818-6.

11. Sarkar, A.; Breitung, B.; Hahn, H. High entropy oxides: The role of entropy, enthalpy and synergy. Scripta Materialia 2020, 187, 43-48. doi:10.1016/j.scriptamat.2020.05.019.

12. Mishra, S.S.; Mukhopadhyay, S.; Yadav, T.P.; Mukhopadhyay, N.K.; Srivastava, O.N. Synthesis and characterization of hexanary Ti-Zr-V-Cr-Ni-Fe high-entropy Laves phase. Journal of Materials Research 2019, 34, 807-818. doi:10.1557/jmr.2018.502.

13. Yadav, T.P.; Mukhopadhyay, S.; Mishra, S.S.; Mukhopadhyay, N.K.; Srivastava, O.N. Synthesis of a single phase of high-entropy Laves intermetallics in the Ti-Zr-V-Cr-Ni equiatomic alloy. Philosophical Magazine Letters 2017, 97, 494-503. doi:10.1080/09500839.2017.1418539.

14. Mishra, S.; Yadav, T.; Srivastava, O.; Mukhopadhyay, N.; Biswas, K. Formation and stability of C14 type Laves phase in multi component high-entropy alloys. Journal of Alloys and Compounds 2020, 832, 153764. doi:10.1016/j.jallcom.2020.153764.

15. Gorban', V.F.; Krapivka, N.A.; Firstov, S.A. High-entropy alloys: Interrelations between electron concentration, phase composition, lattice parameter, and properties. Physics of Metals and Metallography 2017, 118, 970-981. doi:10.1134/S0031918X17080051.

16. Park, H.J.; Na, Y.S.; Hong, S.H.; Kim, J.T.; Kim, Y.S.; Lim, K.R.; Park, J.M.; Kim, K.B. Phase evolution, microstructure and mechanical properties of equi-atomic substituted TiZrHfNiCu and TiZrHfNiCuM $(\mathrm{M}=\mathrm{Co}, \mathrm{Nb})$ high-entropy alloys. Metals and Materials International 2016, 22, 551-556. doi:10.1007/s12540-016-6034-5.

17. Kao, Y.F.; Chen, S.K.; Sheu, J.H.; Lin, J.T.; Lin, W.E.; Yeh, J.W.; Lin, S.J.; Liou, T.H.; Wang, C.W. Hydrogen storage properties of multi-principal-component CoFeMnTixVyZrz alloys. International Journal of Hydrogen Energy 2010, 35, 9046-9059. doi:10.1016/j.ijhydene.2010.06.012.

18. Uporov, S.; Ryltsev, R.; Estemirova, S.; Sterkhov, E.; Chtchelkatchev, N. Stable high-entropy TiZrHfNbVCrMoMnFeCoNiAl Laves phase. Scripta Materialia 2021, 193, 108-111. doi:https:/ / doi.org/10.1016/j.scriptamat.2020.10.049.

19. Yurchenko, N.; Stepanov, N.; Salishchev, G. Laves-phase formation criterion for high-entropy alloys. Materials Science and Technology 2017, 33, 17-22. doi:10.1080/02670836.2016.1153277.

20. Zhu, J.H.; Liu, C.T.; Pike, L.M.; Liaw, P.K. A thermodynamic interpretation of the size-ratio limits for laves phase formation. Metallurgical and Materials Transactions A 1999, 30, 1449-1452. doi:10.1007/s11661-999-0292-5. 
21. Toby, B.H. EXPGUI, a graphical user interface for GSAS. Journal of Applied Crystallography 2001, 34, 210-213. doi:10.1107/S0021889801002242

22. Williamson, G.; Hall, W. X-ray line broadening from filed aluminium and wolfram. Acta Metallurgica $1953,1,22$ - 31. doi:https://doi.org/10.1016/0001-6160(53)90006-6.

23. Ohba, T.; Kitano, Y.; Komura, Y. The charge-density study of the Laves phases, MgZn2 and MgCu2. Acta Crystallographica Section C 1984, 40,1-5, [https:/ / onlinelibrary.wiley.com/doi/pdf/10.1107/S0108270184002791]. doi:10.1107/S0108270184002791.

24. Stein, F.; Leineweber, A. Laves phases: a review of their functional and structural applications and an improved fundamental understanding of stability and properties. Journal of Materials Science 2021, 56, 5321-5427. doi:10.1007/s10853-020-05509-2.

25. Coey, J.M. Magnetism and magnetic materials; Cambridge university press, 2010.

26. Kresse, G.; Furthmuller, J. Efficiency of ab-initio total energy calculations for metals and semiconductors using a plane-wave basis set. Computational Materials Science 1996, 6, 15 - 50. doi:https:/ / doi.org/10.1016/0927-0256(96)00008-0.

27. Perdew, J.P.; Chevary, J.A.; Vosko, S.H.; Jackson, K.A.; Pederson, M.R.; Singh, D.J.; Fiolhais, C. Atoms, molecules, solids, and surfaces: Applications of the generalized gradient approximation for exchange and correlation. Phys. Rev. B 1992, 46, 6671-6687. doi:10.1103/PhysRevB.46.6671.

28. Perdew, J.P.; Wang, Y. Accurate and simple analytic representation of the electron-gas correlation energy. Phys. Rev. B 1992, 45, 13244-13249. doi:10.1103/PhysRevB.45.13244.

29. Kresse, G.; Joubert, D. From ultrasoft pseudopotentials to the projector augmented-wave method. Phys. Rev. B 1999, 59, $1758-1775$. doi:10.1103/PhysRevB.59.1758.

30. Zunger, A.; Wei, S.H.; Ferreira, L.G.; Bernard, J.E. Special quasirandom structures. Phys. Rev. Lett. 1990, 65, 353-356. doi:10.1103/PhysRevLett.65.353.

31. van de Walle, A.; Tiwary, P.; de Jong, M.; Olmsted, D.; Asta, M.; Dick, A.; Shin, D.; Wang, Y.; Chen, L.Q.; Liu, Z.K. Efficient stochastic generation of special quasirandom structures. Calphad 2013, 42, 13-18. doi:10.1016/j.calphad.2013.06.006.

32. Oganov, A.R.; Glass, C.W. Crystal structure prediction using ab initio evolutionary techniques: Principles and applications. J. Chem. Phys. 2006, 124, 244704. doi:10.1063/1.2210932.

33. Oganov, A.R.; Valle, M. How to quantify energy landscapes of solids. J. Chem. Phys. 2009, 130, 104504. doi:10.1063/1.3079326.

34. Kamaeva, L.; Ryltsev, R.; Lad'yanov, V.; Chtchelkatchev, N. Viscosity, undercoolability and short-range order in quasicrystalforming Al-Cu-Fe melts. J. Mol. Liq. 2020, 299, 112207. doi:https:/ / doi.org/10.1016/j.molliq.2019.112207.

35. Lindquist, B.A.; Jadrich, R.B.; Truskett, T.M. Communication: From close-packed to topologically close-packed: Formation of Laves phases in moderately polydisperse hard-sphere mixtures. J. Chem. Phys. 2018, 148, 191101. doi:10.1063/1.5028279. 